\title{
Nonviral gene therapy in vivo with PAM-RG4/ apoptin as a potential brain tumor therapeutic
}

\author{
This article was published in the following Dove Press journal: \\ International Journal of Nanomedicine \\ 23 February 2013 \\ Number of times this article has been viewed
}

\author{
Songhie An* \\ Kihoon Nam* \\ Sunghyun Choi \\ Cheng Z Bai \\ Yan Lee \\ Jong-Sang Park \\ Department of Chemistry, Seoul \\ National University, Seoul, \\ Republic of Korea \\ *These authors contributed equally \\ to this work
}

Correspondence: Jong-Sang Park Department of Chemistry, Seoul National University, 599 Gwanak-ro, Gwanak-gu, Seoul, I5 I-747, Republic of Korea

$\mathrm{Tel}+822$ 87I 5355

Fax +8228775110

Email pjjspark@plaza.snu.ac.kr

Yan Lee

Department of Chemistry, Seoul National University, 599 Gwanak-ro, Gwanak-gu,

Seoul, I5I-747, Republic of Korea

Tel +8228804344

Fax +822 87I 2496

Email gacn@snu.ac.kr
Background: Glioma is still one of the most complicated forms of brain tumor to remove completely due to its location and the lack of an efficient means to specifically eliminate tumor cells. For these reasons, this study has examined the effectiveness of a nonviral gene therapy approach utilizing a tumor-selective killer gene on a brain tumor xenograft model.

Methods and results: The therapeutic apoptin gene was recombined into the JDK plasmid and delivered into human brain tumor cells (U87MG) by using a polyamidoamine dendrimer with an arginine surface (PAM-RG4). Studies in vitro showed that the PAM-RG4/apoptin plasmid polyplex exhibited a particularly high transfection activity of $>40 \%$. Terminal deoxynucleotidyl transferase dUTP nick end labeling (TUNEL) assay, 4',6-Diamidino-2-phenylindole (DAPI) TUNEL assay, DAPI staining, and caspase-3 activity assay verified that the tumor cells had undergone apoptosis induced by apoptin. For in vivo studies, the polyplex was injected into tumors, which were induced by injecting U87MG cells intradermally into nude mice. Based on hematoxylin and eosin staining, epidermal growth factor receptor immunohistochemistry results and tumor volume measurement results, tumor growth was effectively inhibited and no specific edema, irritation, or other harm to the skin was observed after polyplex injection. The in vivo expression of apoptin and the induction of apoptosis were verified by reverse-transcription polymerase chain reaction analysis, TUNEL assay, and DAPI staining.

Conclusion: The PAM-RG4/apoptin gene polyplex is a strong candidate for brain tumor therapeutics because of the synergistic effect of the carrier's high transfection efficiency $(35 \%-40 \%)$ in glioma cells and the selective apoptosis-inducing activity of apoptin in tumor cells.

Keywords: apoptin, PAM-RG4, malignant glioma, nonviral gene therapy, apoptosis

\section{Introduction}

Malignant glioma is a highly lethal form of brain tumor. Despite recent technical advances in surgery and other adjuvant therapies, including chemotherapy and radiotherapy, the recurrence of these tumors appears to be inevitable. Furthermore, the strong infiltrative and highly proliferative properties of brain tumors and their location make complete surgical resection almost impossible. ${ }^{1}$ For these reasons, brain tumors have been one of the earliest targets of gene therapy; ${ }^{2}$ however, although such therapeutic approaches (as adjuvant methods) have been investigated for their promising and novel research results, to date, available treatment methods have only demonstrated modest effects and the field still lacks the combination of a powerful therapeutic gene and an efficient delivery system. ${ }^{3}$

Studies and clinical trials on the treatment of brain tumors with gene therapy have mainly utilized viral vectors. ${ }^{3,4}$ The efficiency of such viral vectors is very high, 
making them an attractive choice; however, in their actual clinical application, side effects and the uncertainty of complete safety from lethal viral characteristics are obstacles to overcome. ${ }^{5}$ Conversely, the application of nonviral carriers as a means of delivering a therapeutic gene to brain tumors has not been achieved in clinical trials, although recently reported biocompatible carriers show low toxicity and high transfection efficiency in vitro and in vivo. ${ }^{6,7}$ PAM-RG4, for example, a polyamidoamine (PAMAM) dendrimer derivative with an arginine surface, is known for its high transfection efficiency, especially in neuronal cells. ${ }^{8}$ Rapidly proliferating glioma cells are therefore attractive targets for this nonviral gene-delivery carrier system. ${ }^{9}$

Among various gene therapy strategies used in the laboratory and clinical trials for brain tumors, ${ }^{10}$ the utilization of tumor-selective killer genes is one of the most promising. Not only would this alleviate the invasiveness, but also enable complete removal of the tumor. One candidate is the apoptin gene, whose mechanism of action has not been clarified yet, but it seems that it activates a series of signals inside the nucleus of a tumor cell, including the phosphorylation of Nur77 and its nuclear export. ${ }^{11}$ In the cytoplasm, Nur77 modulates Bcl-2 proteins, eg, by converting them from antiapoptotic to proapoptotic molecules. ${ }^{11,12}$ This and additional events might induce apoptosis through the mitochondrial pathway of caspase activation.

Although the therapeutic potential of apoptin has been reported for several years, somehow, its therapeutic application is still limited. ${ }^{13}$ Therefore, the present study focused on the development of a treatment for brain tumors by combining the advantages of the apoptin gene and the nonviral PAMRG4 delivery system to create a synergistic effect.

\section{Materials and methods}

\section{Materials}

PAMAM G4 (Starburst), 3-[4,5-dimethylthiazol-2-yl]-2,5diphenyl tetrazolium bromide (MTT), piperidine, $N, N$-dimethylformamide, $N, N$-diisopropylethylamine, polyethylenimine (PEI), and colorimetric caspase-3 assay kit were purchased from Sigma-Aldrich, St Louis, MO, USA. $N$-hydroxybenzotriazole was purchased from Anaspec (San Jose, CA, USA) and Fmoc-1-Arg(pbf)-OH was from Novabiochem (San Diego, CA, USA). ExGen500 was from Fermentas Co, (Waltham, MA, USA), DeadEnd ${ }^{\text {TM }}$ Colorimetric TUNEL System and DeadEnd $^{\mathrm{TM}}$ Fluorometric TUNEL System were both purchased from Promega (Madison, WI, USA). Fetal bovine serum (FBS), trypsin/EDTA and Dulbecco's modified Eagle's medium (DMEM) were purchased from GIBCO
(Gaithersburg, MD, USA). Phosphate-buffered saline (PBS) was from Lonza (Walkersville, MP, USA).

\section{Methods}

\section{Synthesis of PAM-RG4}

PAM-RG4 was synthesized as described in our previous study. ${ }^{14}$ Briefly, amino acid coupling to PAMAM was performed in anhydrous DMF for $4 \mathrm{~h}$ at room temperature with 4 equivalents of HOBt, HBTU, Fmoc-Arg(pbf)-OH and 7.3 equivalent of DIPEA, respectively. The product was precipitated in ethyl ether and washed with excess ether followed by the removal of Fmoc and pbf groups. The final product was precipitated, washed, dialyzed and finally collected after freeze-drying, yielding white powder.

\section{Construction of pJDK-apoptin plasmid DNA (pDNA)} Described in the Supplementary Information.

\section{Formation of polymer/pDNA complexes}

Branched poly(ethyleneimine) (25 kDa; PEI) and ExGen500 were used as the control transfection reagents. Each polyplex was formulated at its optimal weight ratio between the polymer and the pDNA, being 1 and 4 for PEI and PAM-RG4 polyplexes, respectively, and $3.3 \mu \mathrm{L}$ (six equivalents) of ExGen 500 per $1 \mu \mathrm{g}$ of DNA. Polyplexes were formed by first diluting the polymer in distilled water to a final concentration of $1 \mathrm{mg} / \mathrm{mL}$. The appropriate mass of polymer solution was then added to Dulbecco's modified Eagle's medium (DMEM) in a total volume of $100 \mu \mathrm{L}$. The polymer solution was added to the diluted DNA solution and vortexed immediately for 10 seconds. The complex solution was then allowed to stand for 10 or 30 minutes at room temperature (RT).

\section{Cell lines and culture}

Human glioblastoma U87MG cells, rat glioma C6 cells, mouse neuroblastoma Neuro2A cells, mouse HT22 hippocampal cells, and mouse NIH-3T3 embryonic fibroblasts were grown in DMEM supplemented with $10 \%$ heat-inactivated FBS at $37^{\circ} \mathrm{C}$ in a humidified atmosphere containing $5 \% \mathrm{CO}_{2}$. The cells were maintained in T75 cell culture flasks and serially subcultured every 4 days by using trypsin/EDTA.

\section{Luciferase assay}

U87MG cells were seeded in 6-well tissue culture plates at a density of $3.0 \times 10^{4}$ cells/well in $2 \mathrm{~mL}$ of DMEM containing $10 \% \mathrm{FBS}$ and incubated in a humidified $5 \% \mathrm{CO}_{2}$ incubator for 24 hours before transfection. The cells were rinsed with PBS to remove any unattached cells and given $1.8 \mathrm{~mL}$ 
of serum-containing medium. To each well $0.2 \mathrm{~mL}$ of the polymer/pJDK-Luci complex was added and the plates were further incubated for 24 or 48 hours at $37^{\circ} \mathrm{C}$ in a humidified $5 \% \mathrm{CO}_{2}$ incubator. After the growth medium was removed, the cells were washed with PBS and lysed for 30 minutes at RT with $900 \mu \mathrm{L}$ of $1 \times$ Reporter Lysis Buffer (Promega). The expression of the luciferase gene in the transfected cells was evaluated using luminescence measurements made with an LB 9507 luminometer (Berthold, Bad Wildbad, Germany).

\section{Microscopic analysis}

U87MG cells were seeded at a density of $3.0 \times 10^{4}$ cells $/$ well on Lab-Tek chamber slides (Nunc, Rochester, NY, USA) and incubated overnight. The plated cells were transfected with polymer/pEGFP-C2 as described above and cultured for further 48 hours. The cells were washed twice with PBS and fixed in a $4 \%$ formaldehyde/PBS solution for 10 minutes, which was then removed. The samples were rinsed twice with PBS, mounted with 4',6-diamidino-2-phenylindole-containing mounting solution (Vectashield, H-1500; Vector, Burlingame, CA, USA) and coverslipped. The slides were examined by differential interference contrast and fluorescence microscopy by using the BX51TRF fluorescence microscope equipped with a BH2-RFL-T3 UV burner and a DP70 charge-coupled device camera (Olympus, Tokyo, Japan).

\section{Fluorescence-activated cell sorter (FACS) analysis} U87MG cells, transfected with pEGFP-C2 polyplexes, were washed with PBS, trypsinized and incubated with 7-aminoactinomycin D (7-AAD) for 10 minutes at RT. The samples were then washed, centrifuged at $1300 \times g$ for 5 minutes, and resuspended in 2\% FBS in PBS. To quantify the expression of green fluorescence protein (GFP), flow cytometry analysis was performed on a FACSCalibur flow cytometer (Becton Dickinson, Franklin Lakes, NJ, USA). The acquired data were analyzed using CellQuest software (Becton Dickinson). In all experiments, dead cells were excluded from the analysis and sorting.

\section{Assessment of cytotoxicity and antitumor activity by using MTT assay}

U87MG cells were seeded in 6-well culture plates at $3.0 \times 10^{4}$ cells/well in $2 \mathrm{~mL}$ of medium and grown overnight to $70 \%-80 \%$ confluence. After rinsing with PBS, fresh serumcontaining medium was added. The cells were treated with $200 \mu \mathrm{L}$ solutions of naked DNA (pJDK-Luci), PAM-RG4, PEI, ExGen500, and each of their polyplexes. After further incubation for 48 hours, cells were exposed to $300 \mu \mathrm{L}$ of filtered MTT stock solution ( $2 \mathrm{mg} / \mathrm{mL}$ in PBS). After incubation for 4 hours at $37^{\circ} \mathrm{C}$, each MTT-containing medium was removed and $500 \mu \mathrm{L}$ of dimethyl sulfoxide (DMSO) were added to dissolve the formazan crystals formed by the living cells. The samples were then transferred to 96-well plates to measure their absorbance at $570 \mathrm{~nm}$ with a microplate reader (Molecular Devices Co, Menlo Park, CA, USA) and SoftMax Pro v5 (Molecular Devices, Sunnyvale, CA, USA) software.

To confirm the tumor-selective killing effect of the apoptin gene, U87MG, NIH-3T3, C6, and Neuro2A cells were seeded in 24 -well culture plates at $6.0 \times 10^{3}$ cells/well in $600 \mu \mathrm{L}$ of medium and grown overnight to adherence. After rinsing with PBS and addition of fresh FBS-containing medium, the cells were treated with $120 \mu \mathrm{L}$ solutions of PAM-RG4/ pJDK-apoptin as the therapeutic gene or PAM-RG4/pJDK polyplexes as the control and incubated for 2 more days following the same MTT assay as described above. For both results, the relative cell viabilities were calculated, expressed as a percentage of the untreated control, and compared.

\section{Brain tumor xenograft in nude mice}

Five-week-old nude mice were kept at $25^{\circ} \mathrm{C}$ in a specificpathogen-free environment, in positive pressure rooms with a standard 12 hour night/day cycle and with filtered and humidified air. At the time of the experiments, the mice were anesthetized with intraperitoneal injections of Zoletil and Rompun and randomly divided into three groups $(n=6)$. The left flanks of the animals were injected intradermally with $1.0 \times 10^{6}$ U87MG cells in $50 \mu \mathrm{L}$, which were left to grow until the tumor diameter reached 3-4 $\mathrm{mm}$. At this time point, the $(+)$ control group, serving as the untreated control, was injected with PBS only. In the gene therapy groups, $50 \mu \mathrm{L}$ of PBS containing a polyplex of $20 \mu \mathrm{g}$ of PAM-RG4 and $5 \mu \mathrm{g}$ of $\mathrm{pJDK}$ or $\mathrm{pJDK}$-apoptin were injected intratumorally. The diameters of the tumors were measured every other day and used to calculate the tumor volume with the formula $\left(w^{2} \times l\right) / 2$, where $w$ is the smallest and $l$ is the largest diameter of the tumor. ${ }^{15}$ The mice were sacrificed 21 days after gene therapy, at which time the tumor size in the control group exceeded $300 \mathrm{~mm}^{3}$. All animal studies were performed in accordance with the Guide for the Care and Use of Laboratory Animals, as approved by the committee on the care and use of animals in research at Seoul National University.

\section{Immunohistochemistry}

The excised tissues were fixed in 4\% paraformaldehyde solution and embedded in paraffin. Five-micrometer serial 
sections were cut from the tissue blocks and floated onto the glass slides, which were deparaffinized in xylene and rehydrated in a graded series of ethanol to deionized water for subsequent analysis.

\section{Hematoxylin and eosin (H\&E) staining}

Sections were stained in Harris hematoxylin solution for 8 minutes, differentiated in $1 \%$ acid alcohol for 30 seconds and blued in $0.2 \%$ ammonia water for 45 seconds to remove unreacted hematoxylin. After rinsing in 95\% alcohol, the slides were counterstained in an eosin-phloxine B solution for 45 seconds. After dehydration in a graded series of ethanol and xylene, the sections were treated with a mounting solution for observation.

\section{Immunostaining for EGFR}

Tris-buffered saline (TBS) with $0.05 \%$ (v/v) Tween 20 was used to wash the slides, which subsequently underwent antigen retrieval in $10 \mathrm{mM}$ Tris buffer ( $1 \mathrm{mM}$ EDTA, $0.05 \%$ (v/v) Tween 20, pH 9.0) overnight at $60^{\circ} \mathrm{C}$. Endogenous peroxidase was blocked by a 30 -minute incubation in a peroxidase-blocking solution. The sections were treated with $10 \%$ normal goat serum for 1 hour to reduce further nonspecific background staining and incubated in a 200:1 dilution of epidermal growth factor receptor (EGFR) antibody overnight at $4{ }^{\circ} \mathrm{C}$. The slides were washed with TBS and treated with goat anti-rabbit IgG for 1 hour and diaminobenzidine (DAB)+ chromogen with substrate buffer for 2 min (Dako REAL ${ }^{\text {TM }}$ EnVision ${ }^{\mathrm{TM}}$ Detection System; Dako, Glostrup, Denmark). After washing with TBS and tap water, the sections were incubated in hematoxylin for counterstaining. The slides were differentiated in $1 \%$ acid alcohol and blued in $0.2 \%$ ammonia water. After dehydration, the sections were treated with a mounting solution for their long-term storage. ${ }^{16-18}$

\section{RT-PCR analysis}

To detect mRNA expression in tissues, the animals were sacrificed and the tumor and skin of the injection site were collected. The tissues were homogenized and total RNA was extracted using the TRIzol ${ }^{\circledR}$ Reagent according to the manufacturer's instructions (Invitrogen, Carlsbad, CA, USA). Reverse transcriptase PCR (RT-PCR) was performed using the OneStep RT-PCR Kit (QIAGEN, Hilden, Germany) with the following manually designed PCR primers: 5'-CCCGAATTCATGAACGCTCTC-3' (forward), 5'-GCCCTCTAGATCACAGTCTTATACGC-3' (reverse) for apoptin and 5'-CGCTGGGTCA-GAAGGACTCCTATG-3' (forward), 5'-CGGAGAAGAGCTATGAGCTGCCTG-3' (reverse) for the $\beta$-actin housekeeping gene.

\section{TUNEL assay and DAPI staining}

Cells at 3.0 $\times 10^{6} \mathrm{U} 87 \mathrm{MG}$ were plated on Lab-Tek ${ }^{\circledR}$ Chamber Slides and incubated in DMEM containing 10\% FBS. After 1 day, the cells were exposed to PAM-RG4/pJDK-apoptin and PAM-RG4/pJDK polyplexes for a further 48 hours and assessed using the DeadEnd ${ }^{\mathrm{TM}}$ Colorimetric TUNEL System (Promega) to verify the process of apoptosis following the given protocol, where the terminal deoxynucleotidyl transferase, recombinant (rTdT) enzyme incorporated biotinylated nucleotide at the $3^{\prime}-\mathrm{OH}$ DNA ends. Horseradish peroxidase-labeled streptavidin was then bound to these biotinylated nucleotides, which were detected using the peroxidase substrate, hydrogen peroxide, and DAB. The cells were rinsed in fresh PBS, mounted in an aqueous medium and observed under a light microscope. Another experimental group was counterstained with DAPI for nuclei analysis.

Tissue sections for in vivo analysis were collected from animals 3 days after PBS, PAM-RG4/pJDK, or PAM-RG4/ pJDK-apoptin injection and immediately paraffinized before further analysis using the DeadEnd ${ }^{\mathrm{TM}}$ Fluorometric TUNEL System (Promega). Following the given protocol, samples were analyzed using fluorescence microscopy to detect the localized green fluorescence (fluorescein-12-dUTP, which was catalytically incorporated at the $3^{\prime}-\mathrm{OH}$ ends of fragmented DNA in apoptotic cells by using the rTdT enzyme) of apoptotic cells and blue fluorescence (DAPI) of cell nuclei.

\section{Caspase activity test}

In a 24 -well plate, $3.0 \times 10^{6} \mathrm{U} 87 \mathrm{MG}$ cells were plated and incubated in DMEM containing 10\% FBS. After 1 day, PAM-RG4/pJDK-apoptin and PAM-RG4/pJDK polyplexes were added to the cells for 48 hours. After collecting the cells, caspase-3 activity was measured in vitro by using a colorimetric caspase-3 assay kit (Sigma, Saint Louis, MO, USA). The collected cells were lysed and centrifuged at $16000 \times g$ for 15 minutes at $4^{\circ} \mathrm{C}$. A 96-well plate with $85 \mu \mathrm{L}$ of $1 \times$ assay buffer and $10 \mu \mathrm{L}$ of the caspase- 3 substrate Ac-DEVD-pNA was added to $5 \mu \mathrm{L}$ of cell lysate and subsequently incubated in a humidified chamber at $37^{\circ} \mathrm{C}$ for 24 hours. The absorbance was measured at $405 \mathrm{~nm}$ by using a microplate reader and recorded as a percentage relative to the cells that were not treated with the polyplexes. 


\section{Statistical analyses}

Statistical analyses were performed using the SPSS program (version 19.0; IBM, Armonk, NY, USA). A Student's $t$-test or analyses of variance was used to analyze differences between groups. All data were presented as mean \pm standard deviation (SD) or mean \pm standard error of the mean (SEM). $P$ values less than 0.05 were considered significant.

\section{Results and discussion}

This study presents a new therapeutic method for the treatment of brain tumors by combining powerful and advantageous approaches for gene therapy, ie, the apoptin gene and the nonviral gene delivery carrier PAM-RG4. In vivo studies showed a significant decrease in the volume of tumors and even their elimination.

\section{Transfection efficiency in vitro}

PAM-RG4 was used to transfect U87MG to assess its transfection efficiency as a possible nonviral carrier for the treatment of malignant glioma. The PAM-RG4/pDNA complex has shown substantially enhanced gene expression levels with low cytotoxicity in various cells making PAM-RG4 a suitable gene delivery carrier candidate for targeting brain tumors. Especially, PAM-RG4 displays excellent transfection efficiency on nerve cells over other commercially available nonviral gene delivery systems, which is presumably because of the arginine residues that are localized on the surface of the dendrimer. Various protein transduction domains, such as TAT, or membrane translocation signals sequences are rich in arginine, which leads to the hypothesis that this particular modification facilitates the cell membrane-penetrating activity of PAM-RG4 during its cellular uptake or nuclear translocalization efficiency after entry into the cytosol. ${ }^{19,20}$ Studies in primary cortical neurons have also shown that PAM-RG4 results in transfection levels of $35 \%-40 \%$, which are significantly higher than those reported for other commercial nonviral gene delivery carriers such as Lipofectamine ${ }^{\mathbb{R}}$ (Life Technologies, Carlsbad, CA, USA), 25-kDa PEI, and native PAMAM. ${ }^{14}$ The tumorigenic nature of U87MG can therefore be used in vivo to establish either subcutaneous or intracerebral tumor models.

PAM-RG4/pDNA polyplexes have already been characterized and their stability assayed in our previous studies, ${ }^{7,8}$ showing that polyplexes maintain the size of about $200 \mathrm{~nm}$ at a charge ratio ranging from 2 to 10 and that with heparin, less than $5 \%$ of the pDNA was released when the heparin/pDNA mole ratio was below 2000 . Therefore, the observed effects can be concluded to be due to the bound DNA-polymer complex.
The transfection capacities of the polyplexes were tested using two types of plasmids containing a reporter gene encoding either firefly luciferase or GFP. Luciferase expression was evaluated after 24 or 48 hours in the presence of serum. Serum proteins are known to interact with polyplexes and inhibit gene transfer, reducing the transfection efficiency, however, in spite of their presence, PAM-RG4 showed a high level of transfection, so serum should not limit its use. The transfection activity profiles after incubation with the polyplex for 24 or 48 hours showed similar tendencies, indicating that PAM-RG4 induces higher gene expression levels than other commercial transfection agents known to have superior gene transfer activity (Figure 1A).

The fluorescent images of the transfected cells shown in the right column confirmed that the fluorescent cell portion was highest in those treated with PAM-RG4 (Figure 1B). The optical microscopy images corresponding to each fluorescent image are shown in the left column. Furthermore, cellular GFP expression measured by FACS clearly supported the previous finding that PAM-RG4 achieved outstanding transfection activity under the same experimental conditions used for the microscopic observations (Figure 1C). Approximately $40 \%$ of cells were GFP-positive, which was a remarkable result relative to the numbers produced with the other nonviral vectors.

\section{Cytotoxicity assay in vitro}

Although diverse strategies have been used for the treatment of malignant gliomas, there has been no breakthrough increasing the long-term survival of patients and none of the currently available methods seems to be totally curative. ${ }^{21}$ Patients with high-grade gliomas face a dismal prognosis and often suffer from the deleterious adverse effects of aggressive multimodal treatments. Despite the formidable and infiltrative nature of gliomas, malignant gliomas also have important features relevant to gene therapy. ${ }^{22}$ Because they occur in the brain, a closed cavity protected by the blood-brain barrier, they usually do not metastasize to other organs, unlike most common forms of cancers. ${ }^{23}$ Although the total removal of the tumor is impossible, surgical resection has always preceded extensive combined modalities that incorporate adjuvant radiotherapy and chemotherapy. In this respect, gene therapy for gliomas may be feasibly applied by a local intracranial injection in association with surgical and stereotactic techniques. Therefore, the most important criterion for an ideal vector for the treatment of malignant glioma is that the carrier must facilitate the expression of the therapeutic gene with high efficacy in a target-specific manner. 


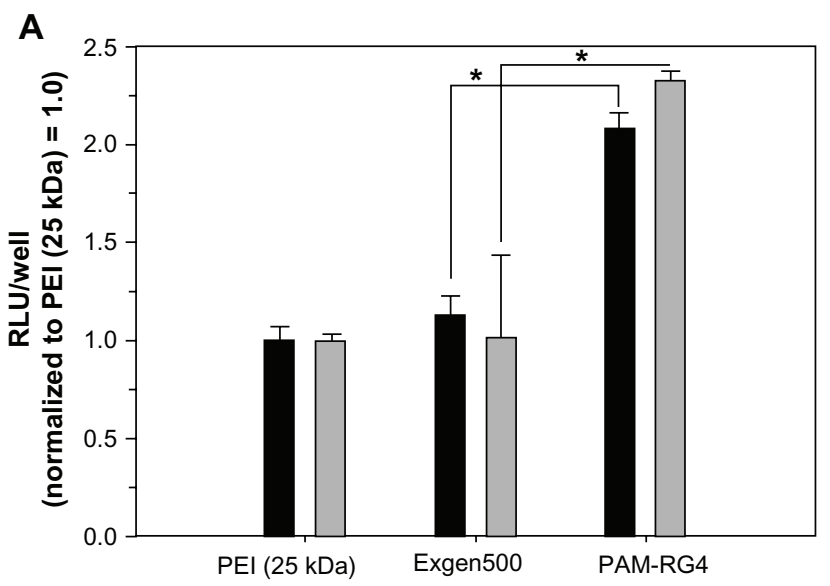

B
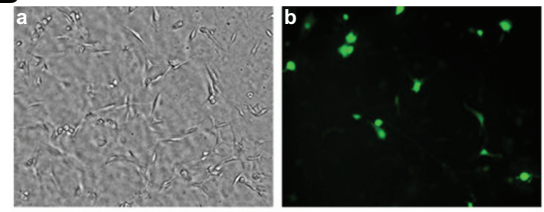

PEI (25 kDa)/ pEGFP-C2
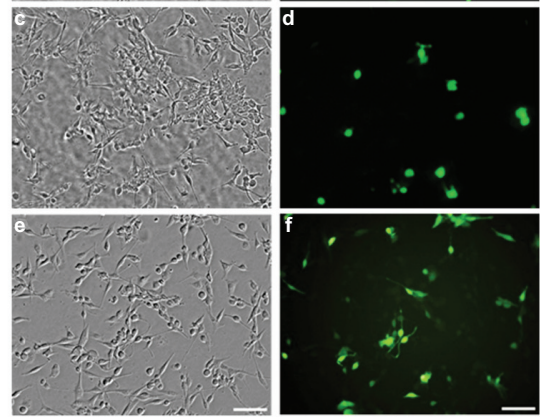

Exgen500/ pEGFP-C2

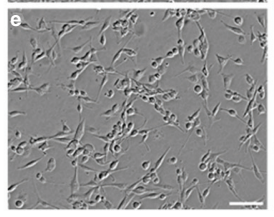

Optical microscopy images

Fluorescence microscopy images
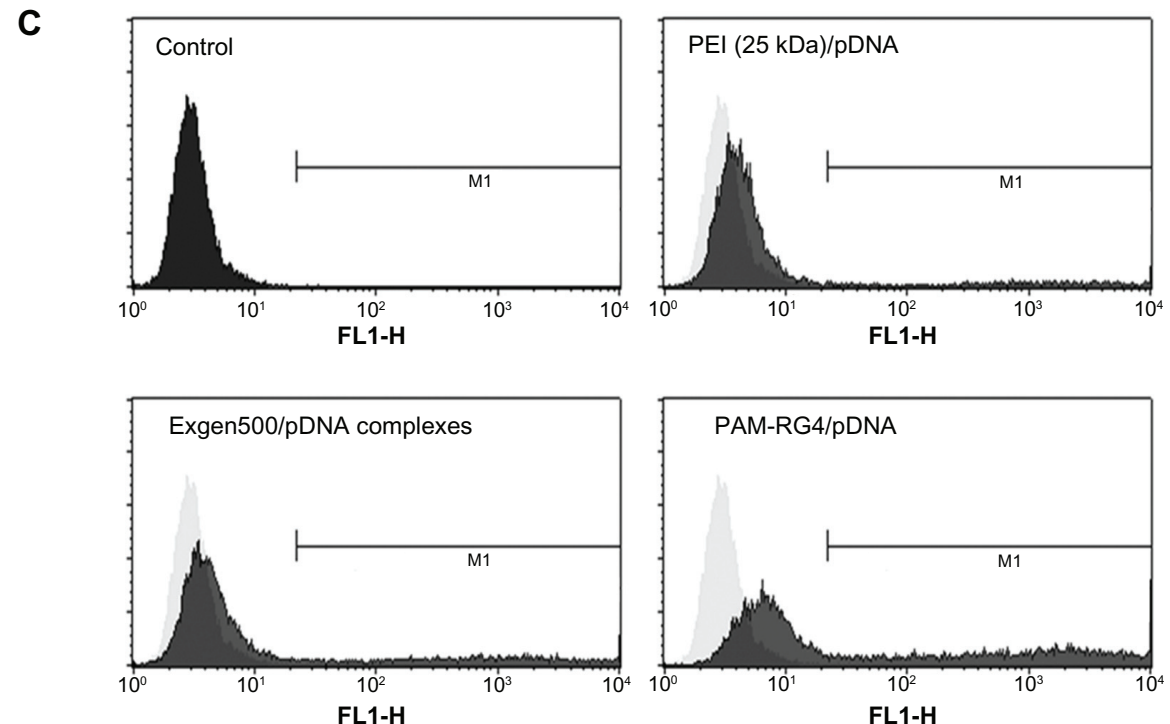

Figure I In vitro transfection efficiency of polyplexes in U87MG cells. Luciferase activity (A). Dark bars for 24 hours and gray bars for 48 hours. All presented data were expressed as mean \pm standard deviation $(n=3)(* P<0.01)$. Fluorescence microscopy images of U87MG cells incubated with the respective complexes $(\mathbf{B})$. Scale bar represents $10 \mu \mathrm{m}$. Cell fluorescence intensity analysis by flow cytometry (C).

Notes: The x-axis represents fluorescence intensity and $y$-axis stands for number of cells. The grey shadow in the three images is the FACS result of the control.

Moreover, it should not have any detrimental adverse effects, such as immunogenicity or cytotoxicity.

The cytotoxicity of PAM-RG4, PEI, and ExGen500 to $\mathrm{U} 87 \mathrm{MG}$ cells at identical transfection concentrations is shown in Figure 2A. The viability of cells treated with only the gene delivery carriers are presented as black bars, showing an approximately $86 \%$ cell viability for PAM-RG4 at a polymer concentration of $4 \mu \mathrm{g} / \mathrm{mL}$, whereas the viability of cells treated with $1 \mu \mathrm{g} / \mathrm{mL}$ PEI or $0.7264 \mu \mathrm{g} / \mathrm{mL}$ ExGen500 was $72 \%$ or $64 \%$, respectively. The cytotoxicity of the polyplexes was examined at the same polymer concentration and the optimum $\mathrm{pJDK} /$ polymer weight ratio for each polyplex and results are presented as grey bars. PAM-RG4 alone and
PAM-RG4/pJDK were much less cytotoxic than PEI and ExGen500. Generally, cell viability tended to decrease with increasing concentrations of the polymers because the accumulation of their enhanced positive charge may be toxic. It is noteworthy that PAM-RG4 treatment allowed the greatest cell viability, although of the three polymers tested, a greater amount of PAM-RG4 was required to achieve maximum efficiency.

\section{Antitumor activity of PAM-RG4/apoptin polyplex in vitro}

Apoptin is a very interesting candidate as an antitumor agent. It reportedly induces the selective death of cell lines 

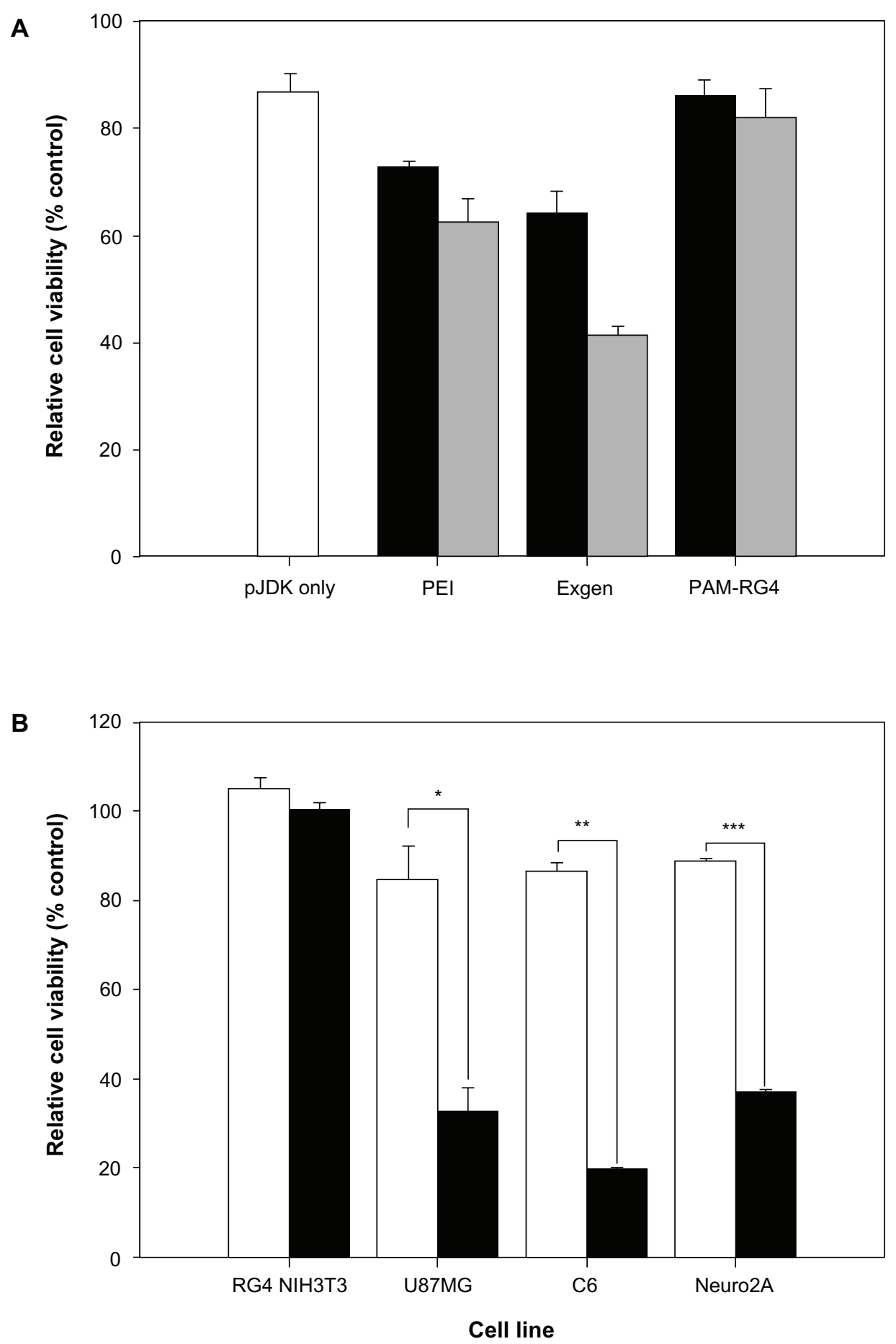

Figure 2 (A) Assessment of cytotoxicity in U87MG cells by using the MTT assay. The black bars represent polymer and the gray bars represent polyplex. (B) The antitumor activity of PAM-RG4/PJDK-apoptin polyplexes on various cell lines by MTT assay.

Notes: The white and black bars represent assay results of PAM-RG4/PJDK and PAM-RG4/PJDK-apoptin, respectively. $* P<0.0$ I; $* * P<0.005 ; * * * P<0.000$ I. All presented data are expressed as the mean \pm standard deviation $(n=3)$.

derived from diverse human tumors. ${ }^{24}$ The apoptotic activity of apoptin combined with PAM-RG4 was estimated with an MTT assay to test the utility of PAM-RG4 as a potent gene delivery system when combined with it (Figure 2B). PAM-RG4/pJDK-apoptin showed a significant cytotoxic effect on tumor cells (U87MG, C6, and Neuro-2A) where relative cell viability was reduced to $30 \%$ after 2 days. On the contrary, NIH-3T3, a normal fibroblast cell line, showed almost the same level of cell viability compared to its control where only PAM-RG4/pJDK was transfected to the cells. 
Such pattern was also observed on the normal neuronal cell line HT22 (Supplementary Information Figure S1).

\section{Antitumor effect PAM-RG4/apoptin polyplex in vivo}

Studies in our xenograft model have shown that the treatment of U87MG tumors with PAM-RG4/pJDK-apoptin significantly reduced the tumor volume to $16 \%$ of the control PBS-treated tumors after 21 days (Figure 3). None of the groups showed any significant tumor growth in the first 10 days. After that time point, however, the tumors grew exponentially in the two control groups and formed an oval-shaped red lump; some to such an extent that the tumor festered (data not shown). On the contrary, in the experimental group, treated with PAM-RG4/pJDK-apoptin, almost no tumor growth was observed.

\section{A}

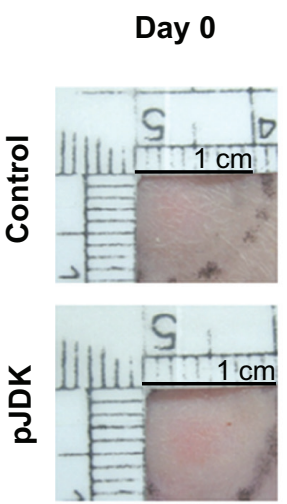

Day 10

Day 21
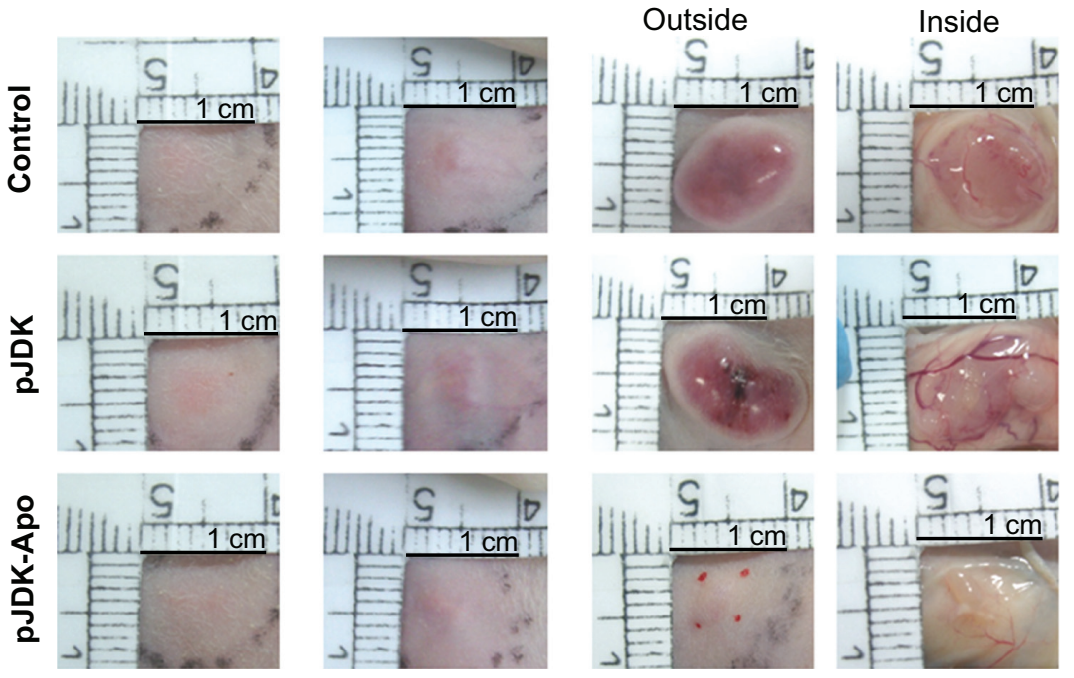

B

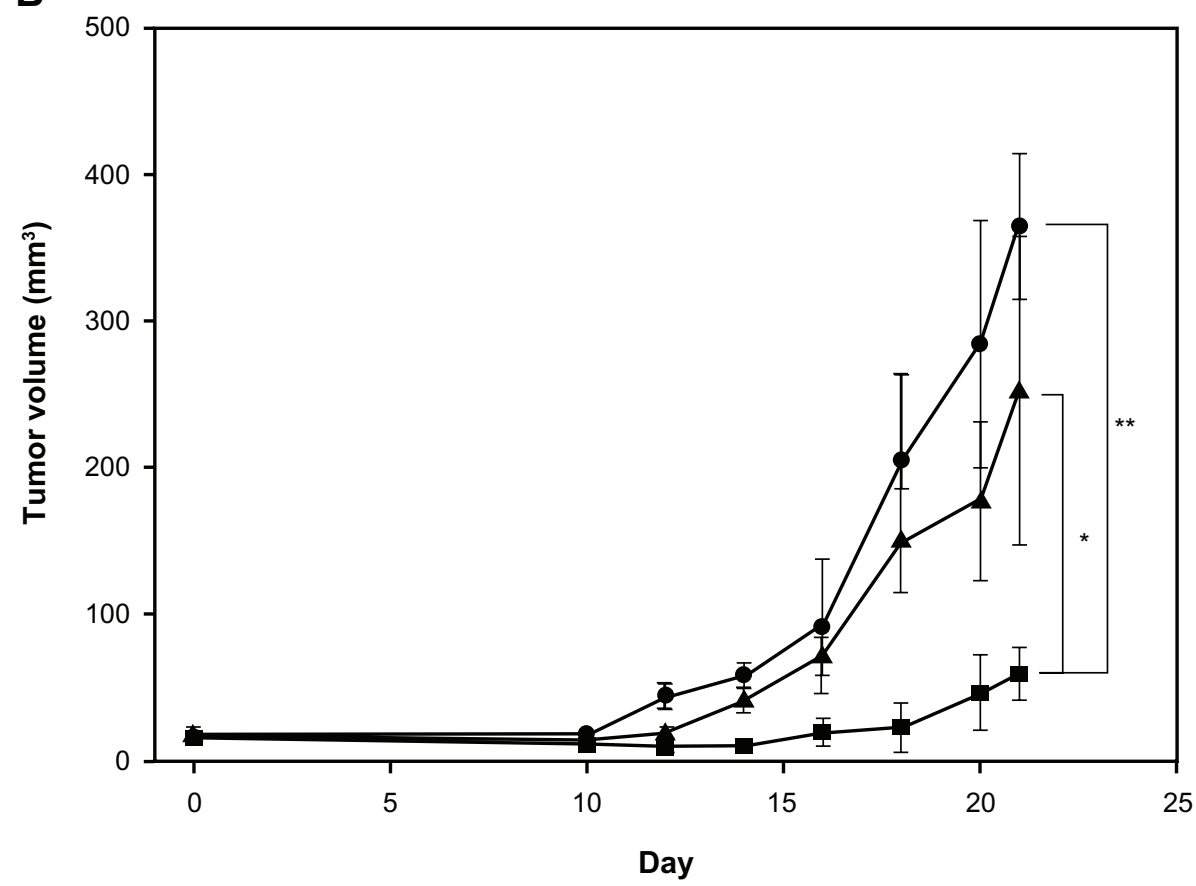

Figure 3 Antitumor activity of PAM-RG4/PJDK-apoptin polyplexes in brain tumor xenografts. Morphology (A) and measured tumor volume (B). Control group (•) and gene therapy group with PAM-RG4/PJDK complexes $(\boldsymbol{\Delta})$ and PAM-RG4/PJDK-apoptin complexes $(\boldsymbol{-})$. The black and red dots are from markers to indicate the injection site. Additional scale bars were added to (A) to improve legibility.

Notes: All presented data are expressed as the mean \pm standard error $(* P<0.0 \mathrm{I} ; * * \mathrm{P}<0.05$ at day $2 \mathrm{I})$. 
As a result, just a single injection of PAM-RG4/pJDKapoptin was shown to be effective for the treatment of brain tumors; however, such an effect is not permanent, as can be inferred from the slightly increasing slope of the volume of tumor that started from $\sim 18$ days after injection (Figure 3 ). In some mice, the tumor started to grow again after $\sim 2.5$ weeks, which could be due to a decrease in apoptin expression. During treatment, the activity of apoptin and tumor growth compete against each other, which results in cancer cell death when apoptin expression is dominant and tumor recurrence when cancer cells grow at a faster rate than apoptosis could be induced. It seems that at around that time point, apoptin expression decreases, leading to the conclusion that for complete treatment, repeated injections will be necessary. Conversely, this observation (of tumor regrowth) could simply represent the growth of cells that weren't transfected with apoptin, as the ones that were transfected should be dead by this time point, rather than a down regulation of apoptin expression. Either hypothesis should be evaluated further. When clinically applied, maximal therapeutic effects could be expected if the PAM-RG4/pJDK-apoptin polyplex would be applied on the remaining glioma cells that are difficult to remove after surgical tumor resection. In addition, Kim et al recently introduced a nasal delivery method using a similar PAMAM derivative, ${ }^{25}$ suggesting that for repeated administration, noninvasive methods can be an option for this polyplex.

To verify the effectiveness of gene therapy, H\&E staining and EGFR immunohistochemical staining of the xenograft brain tumor were performed (Figure 4). H\&E stains the nucleus purple and the cytoplasm red, which allows for the observation of the overall cell organization and form. Such staining (groups A, C, E) showed the inserted tumor between the epidermis and the substructure in the two control groups $(\mathbf{A}, \mathbf{C})$. Also, EGFR is overexpressed in a number of gliomas, but not on normal brain cells. ${ }^{26-30}$ Therefore, we expected a difference in the EGFR-staining intensity between the PAM-RG4/pJDK-apoptin-treated group and the control groups, because if apoptin was actually active, less glioma cells had to be present in the therapeutic group leading to weaker EGFR-staining intensity compared to the control groups. Indeed, strong EGFR-staining intensity can be easily observed in the control groups $\mathbf{B}$ and $\mathbf{D}$, but not in the therapeutic group,
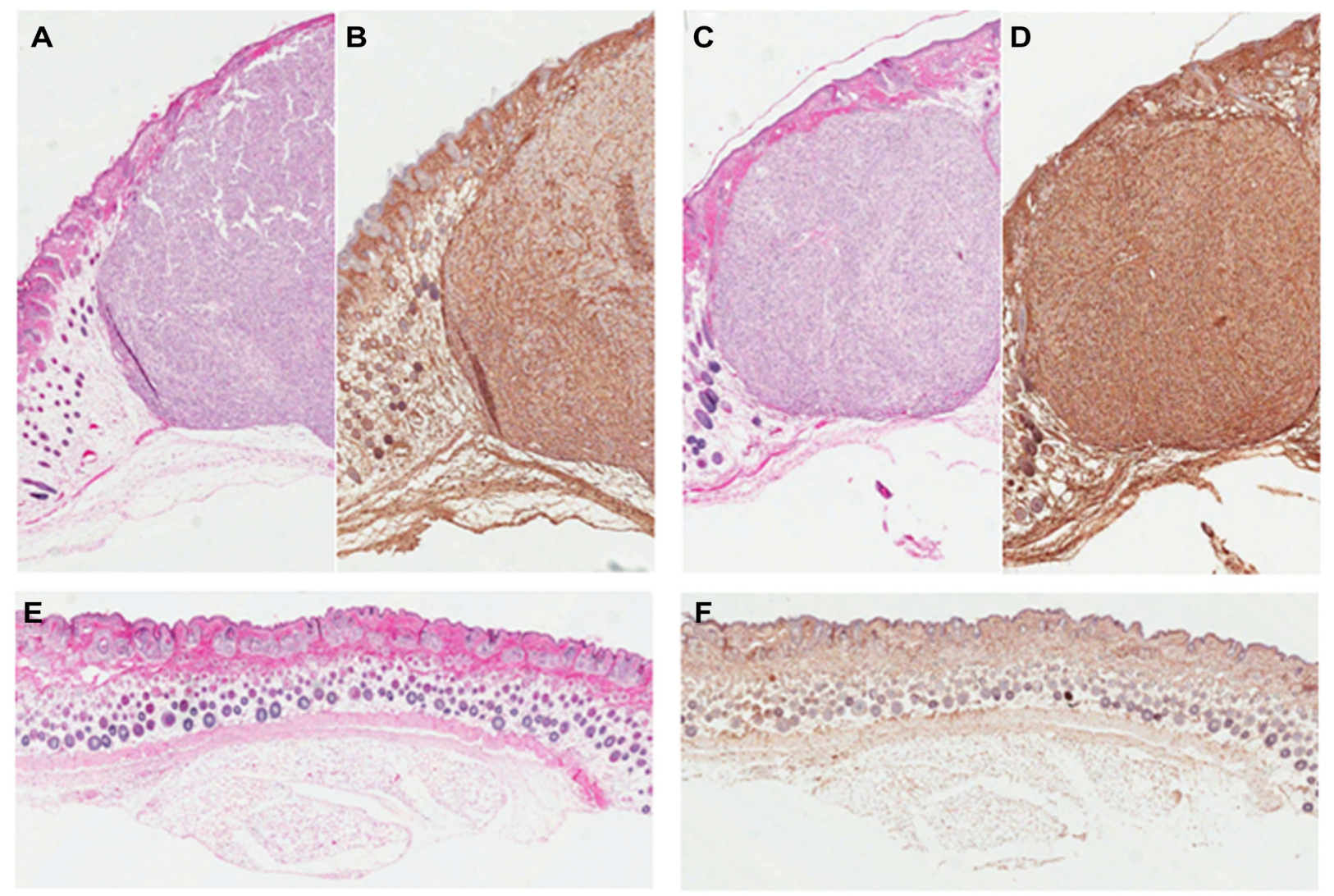

Figure 4 Hematoxylin and eosin staining (A, C and E) and immunostaining for EGFR (B, D and F). Control group (A and B) and gene therapy group with PAM-RG4/pJDK complexes (C and $\mathbf{D})$ and PAM-RG4/PJDK-apoptin complexes (E and $\mathbf{F})$. 
F. Since there are no correlations between apoptin and EGFR expression levels, ${ }^{31}$ this can be inferred as a result of a decrease in the number of glioma cells, due to the apoptotic effect of apoptin. Although a small lump was observed in the PAM-RG4/pJDK-apoptin-treated group (Figure 3A), no signs of tumor could be observed according to the $H \& E$ staining results $(\mathbf{E})$, where the two outer dermal layers were tightly bound to each other and converged well, and the EGFR staining results (F), where strong binding of the EGFR antibody was absent.

\section{Verification of apoptosis}

To verify whether the tumor cell-specific cytotoxicity (Figure 2B) and the prevention of tumor growth (Figure 3) were caused by apoptosis induced by apoptin, various apoptosis assay methods were performed in vitro and in vivo. According to TUNEL assay results (Figure 5A-C), U87MG cells treated with only PBS or PAM-RG4/pJDK (Figure $5 \mathrm{~A}$ and B) preserved their healthy forms, whereas dark brown spots, as indicated with arrows, were observed in the PAM-RG4/pJDK-apoptin treated group (Figure 5C), which indicate apoptosis. This observation was also supported by DAPI staining, where the nucleus is stained and observed as a blue spot (Figure $5 \mathrm{E}$ and F). Healthy cells are round, whereas apoptosis is manifested by cytoplasmic and nuclear condensation, nuclear fragmentation, etc. This differs from necrosis, which is, for example, evinced by cytoplasmic swelling, some condensation of nuclear chromatin, etc. $^{32}$ Treatment with PAM-RG4/pJDK-apoptin $(\mathbf{F})$, in comparison with the PAM-RG4/pJDK-treated group (E), resulted in the characteristic fluorescence of apoptotic cells, indicating
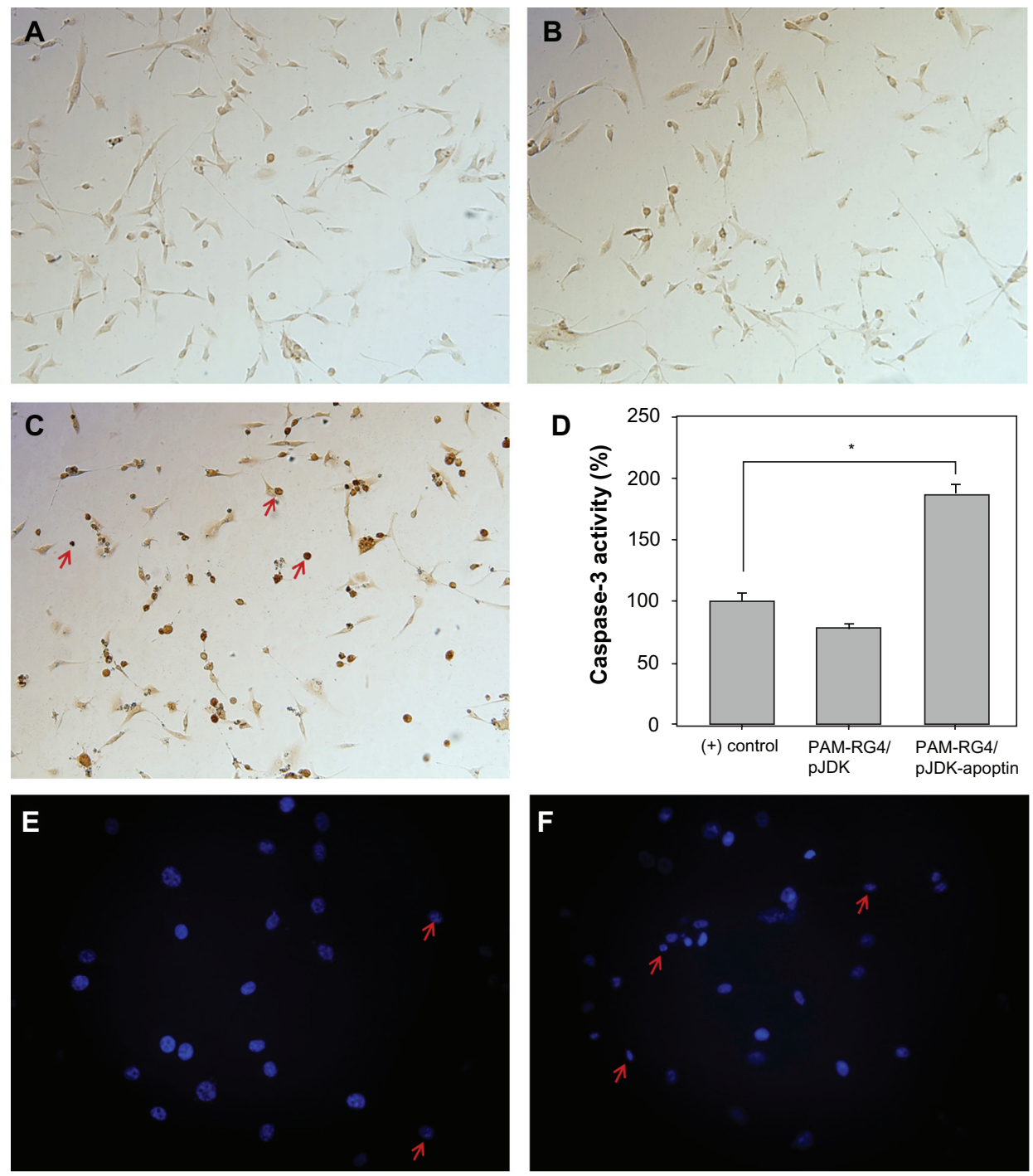

Figure 5 Comparison of apoptosis by using the TUNEL assay for control (phosphate-buffered saline) (A), PAM-RG4/PJDK (B), and PAM-RG4/PJDK-apoptin polyplex (C). Comparison of caspase-3 activity of PAM-RG4/PJDK-apoptin in vitro (D) (*P $<0.01$ ). The DAPI test results for the PAM-RG4/PJDK polyplex (E) and PAM-RG4/PJDK-apoptin polyplex (F) Apoptotic cells are indicated by arrows in $(\mathbf{C})$ and $(\mathbf{F})$. All presented data are expressed as the mean \pm standard deviation. 
DNA condensation (indicated with arrows), in addition to a twofold higher caspase-3 activity, another indicator of apoptosis (Figure 5D).

In this study, PAM-RG4 mediated the transfer of the apoptin gene and induced the apoptosis of tumor cells. Studies have shown that apoptin activates caspase-3, a cysteine protease that catalyzes several executional stages of the apoptotic pathway. ${ }^{33}$ Our study on caspase-3 activity is consistent with such studies (Figure 5D). Caspase-3 activity may also be enhanced by the Fas apoptotic pathway, since apoptin interacts with the Fas-associated death domain (FADD) protein. It is reported that upon its overexpression, apoptin colocalizes with FADD, a component of the death receptor pathway, in so-called death-effector filaments. Other factors such as cell division time were also reported to be crucial. ${ }^{34}$ The rationale is that an increased rate of protein (ie, apoptin) synthesis in fast growing cells induces apoptosis earlier since the critical concentration of apoptin is achieved faster. The cell lines used in this study have doubling times that vary from $\sim 22$ hours (HT22 and NIH-3T3) to longer than 30 hours (U87MG and Neuro2A). Given the fact that normal cell lines such as HT22 and NIH-3T3 grow faster than the tumorigenic U87MG, but showed significantly higher cell viability against transfected apoptin (Figure 2B), the tumor-specific induction of apoptosis by apoptin seems to depend on a more essential factor. The increased activity of caspase- 3 is known to activate caspase-activated DNase by cleaving its inhibitor to rapidly fragment nuclear DNA.

For in vivo analysis, all samples were taken 3 days after their respective treatment. Total RNA was isolated from the tissues and analyzed by RT-PCR. All three samples were positive for the $\beta$-actin housekeeping gene (Figure 6A), but apoptin was only expressed in the PAM-RG4/pJDK-apoptin treated, ie, the therapeutic group. Both of the control samples were negative for apoptin. We also observed further evidence of apoptosis, chromatin condensation, which is caused by the degradation of nuclear membrane proteins, by using DAPI staining. The TUNEL assay results in vitro show significantly more condensed, dark brown spots, indicating that the experimental group had undergone DNA fragmentation as a result of apoptin-induced apoptosis (Figure 5C). The PAM-RG4/ pJDK-treated group also presented a slight degree of DNA fragmentation, which could be due to the slight cytotoxicity of PAM-RG4 (Figure 5E). Such an observation may cause controversy and safety concerns; however, when applied in vivo, the experimental group did not show any signs of side effects, indicating a sufficiently low level of cytotoxicity,
A

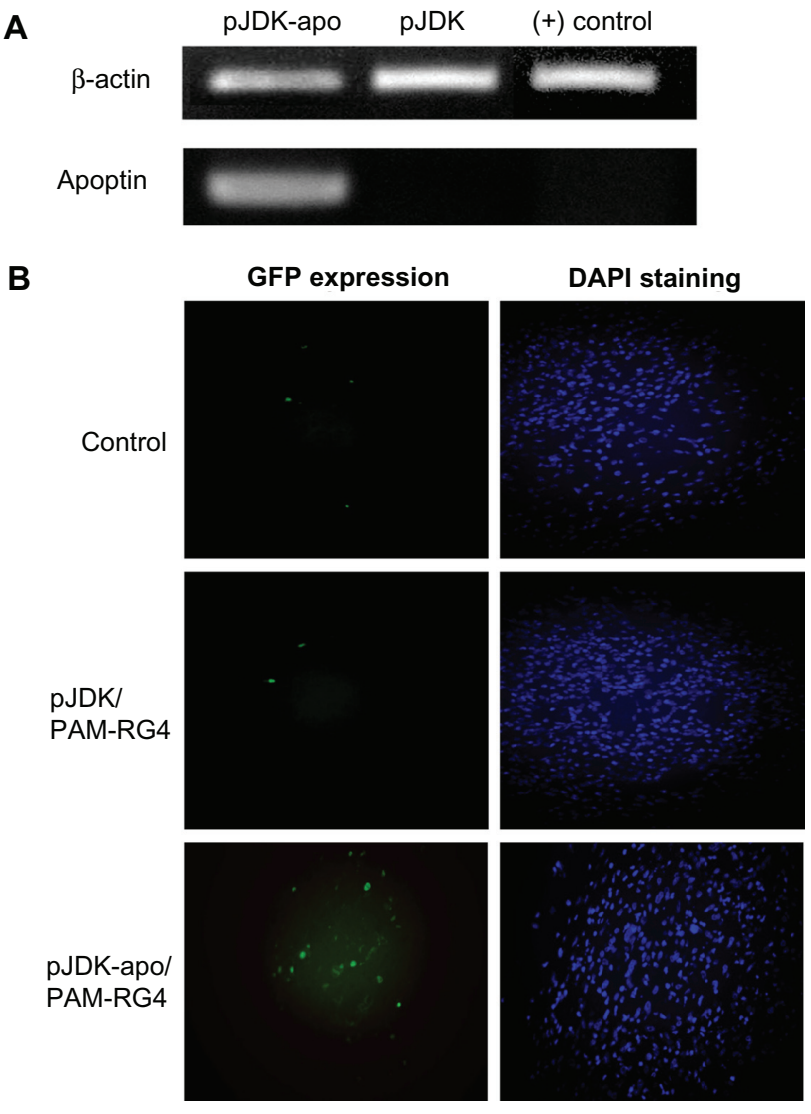

Figure 6 RT-PCR analysis (A) and TUNEL assay and DAPI test (left and right columns of (B), respectively) of the in vivo samples.

Note: PAM-RG4 was used as the gene delivery carrier for pJDK-apoptin and PJDK.

Abbreviation: RT-PCR, reverse transcription polymerase chain reaction.

although it showed similar DAPI and TUNEL assay results (Figure 6B).

According to TUNEL assay results (Figure 6B) on the left, the control and PAM-RG4/pJDK-treated groups exhibited only few green fluorescence spots, whereas the PAMRG4/pJDK-apoptin-treated group showed that apoptosis is active on the third day after it was injected. DAPI-stained images on the right of the figure show the whole distribution of cells. It can be inferred from these results that, as time goes by, the amount of apoptotic cells will increase due to the action of apoptin.

\section{Conclusion}

The PAM-RG4/pJDK-apoptin polyplex had an apoptotic effect, specifically on tumor cells, and did not show any adverse effects, in a brain tumor xenograft. It may be still an early stage to conclude these results to be successful tumor targeting gene therapy since the injections of the polyplex are intratumoral. However, at the same time, this research stresses that this polyplex can be assumed to have a potential 
role in tumor-targeting gene therapy for malignant gliomas. Further research is required in brain orthotopic tumor models to determine the tumor-specific apoptotic effects of our polyplex in the actual target environment. In addition, a systemic administration method of the polyplex has to be studied to accomplish true tumor targeting. The mechanism of apoptin should also be studied further to find the most appropriate application for the successful gene therapy of malignant gliomas.

\section{Acknowledgments}

We thank Professor Marek Los (Interfaculty Institute for Biochemistry, University of Tübingen, Germany) for his kind gift, pEGFP-C1-apoptin and Dr Duk Kyung Kim (Samsung Medical Center, Sunkyunkwan University, Republic of Korea) for kindly providing the pJDK vector. This study was supported by a grant from the Gene Therapy Project of the Ministry of Education, Science and Technology in the Republic of Korea (20110018684) and the Basic Science Research Program through a National Research Foundation of Korea (NRF) grant funded by the Korean government (2012 K001411).

\section{Disclosure}

The authors declare no conflict of interest.

\section{References}

1. Bernstein M, Berger MS. Neuro-oncology: the essentials, 2nd ed. New York, NY: Thieme; 2008.

2. Rainov NG, Ren H. Gene therapy for human malignant brain tumors. Cancer J. 2003;9(3):180-188.

3. Wakabayashi T, Iwami K, Natsume A. Gene therapy for high-grade glioma. Neurol Med-Chir. 2010;50(9):727-736.

4. Candolfi M, Xiong W, Yagiz K, et al. Gene therapy-mediated delivery of targeted cytotoxins for glioma therapeutics. Proc Natl Acad Sci USA. 2010;107(46):20021-20026.

5. Sheridan C. Gene therapy finds its niche (2011;29:121). Nat Biotechnol. 2011;29(5):459-459.

6. Lee JK, Kim ID, Lim CM, et al. Neuroprotection by biodegradable PAMAM ester (e-PAM-R)-mediated HMGB1 siRNA delivery in primary cortical cultures and in the postischemic brain. J Control Release. 2010;142(3):422-430.

7. Park JS, Nam HY, Hahn HJ, et al. Evaluation of generations 2, 3 and 4 arginine modified PAMAM dendrimers for gene delivery. Int J Pharm. 2008;363(1-2):199-205.

8. Park JS, Kim TI, Bai CZ, Nam K. Comparison between arginine conjugated PAMAM dendrimers with structural diversity for gene delivery systems. J Control Release. 2009;136(2):132-139.

9. Sheridan C. Gene therapy finds its niche. Nat Biotechnol. 2011;29(2): 121-128.

10. Engelhard HH. Gene therapy for brain tumors: The fundamentals. Surg Neurol. 2000;54(1):3-9.

11. Maddika S, Booy EP, Johar D, Gibson SB, Ghavami S, Los M. Cancerspecific toxicity of apoptin is independent of death receptors but involves the loss of mitochondrial membrane potential and the release of mitochondrial cell-death mediators by a Nur77-dependent pathway. J Cell Sci. 2005;118(19):4485-4493.
12. Lin BZ, Kolluri SK, Lin F, et al. Conversion of Bcl-2 from protector to killer by interaction with nuclear orphan receptor Nur77/TR3. Cell. 2004;116(4):527-540.

13. Qu S, Sun J, Yan Y, et al. PTD4-apoptin protein therapy inhibits tumor growth in vivo. Int J Cancer. 2009;124(12):2973-2981.

14. Choi JS, Nam K, Park JY, Kim JB, Lee JK, Park JS. Enhanced transfection efficiency of PAMAM dendrimer by surface modification with L-arginine. J Control Release. 2004;99(3):445-456.

15. Engebraaten O, Hjortland GO, Juell S, Hirschberg H, Fodstad O. Intratumoral immunotoxin treatment of human malignant brain tumors in immunodeficient animals. Int J Cancer. 2002;97(6):846-852.

16. Gorgoulis V, Aninos D, Mikou P, et al. Expression of EGF, TGFalpha and EGFR in squamous cell lung carcinomas. Anticancer Res. 1992;12(4):1183-1188.

17. Berger MS, Gullick WJ, Greenfield C, Evans S, Addis BJ, Waterfield MD. Epidermal growth-factor receptors in lung-tumors. J Pathol. 1987;152(4):297-307.

18. Siegal GP, Wang D, Stockard CR, et al. Immunohistochemistry in the evaluation of neovascularization in tumor xenografts. Biotech Histochem. 2008;83(3-4):179-189.

19. Niidome T, Okuda T, Sugiyama A, Aoyagi H. Characters of dendritic poly((L)-lysine) analogues with the terminal lysines replaced with arginines and histidines as gene carriers in vitro. Biomaterials. 2004;25(3):537-544.

20. Fischer D, Li YX, Ahlemeyer B, Krieglstein J, Kissel T. In vitro cytotoxicity testing of polycations: influence of polymer structure on cell viability and hemolysis. Biomaterials. 2003;24(7):1121-1131.

21. Muller H, Brock M, Ernst H. Long-term survival and recurrence-free interval in combined surgical, radiotherapy and chemotherapy of malignant brain gliomas. Clin Neurol Neurosur. 1985;87(3):167-171.

22. Oldfield EH, Ram Z, Culver KW, et al. Therapy of malignant brain tumors by intratumoral implantation of retroviral vector-producing cells. Nat Med. 1997;3(12):1354-1361.

23. Begley DJ. Delivery of therapeutic agents to the central nervous system: the problems and the possibilities. Pharmacol Therapeut. 2004;104(1):29-45.

24. Los M, Panigrahi S, Rashedi I, et al. Apoptin, a tumor-selective killer. Biochim Biophys Acta. 2009;1793(8):1335-1342.

25. Kim ID, Shin JH, Kim SW, et al. Intranasal delivery of HMGB1 siRNA confers target gene knockdown and robust neuroprotection in the postischemic brain. Mol Ther. 2012;20(4):829-839.

26. Miller CR, Buchsbaum DJ, Reynolds PN, et al. Differential susceptibility of primary and established human glioma cells to adenovirus infection: Targeting via the epidermal growth factor receptor achieves fiber receptor-independent gene transfer. Cancer Res. 1998;58(24): 5738-5748.

27. Wikstrand CJ, McLendon RE, Friedman AH, Bigner DD. Cell surface localization and density of the tumor-associated variant of the epidermal growth factor receptor, EGFRvIII. Cancer Res. 1997;57(18): $4130-4140$.

28. Libermann TA, Nusbaum HR, Razon N, et al. Amplification, enhanced expression and possible rearrangement of EGF receptor gene in primary human-brain tumors of glial origin. Nature. 1985;313(5998): 144-147.

29. Libermann TA, Razon N, Bartal AD, Yarden Y, Schlessinger J, Soreq H. Expression of epidermal growth factor receptors in human brain tumors. Cancer Res. 1984;44(2):753-760.

30. Wong AJ, Bigner SH, Bigner DD, Kinzler KW, Hamilton SR, Vogelstein B. Increased expression of the epidermal growth-factor receptor gene in malignant gliomas is invariably associated with gene amplification. Proc Natl Acad Sci U S A. 1987;84(19):6899-6903.

31. Argiris K, Panethymitaki C, Tavassoli M. Naturally occurring, tumor-specific, therapeutic proteins. Exp Biol Med. 2011;236(5): 524-536.

32. Seralini GE, Benachour N. Glyphosate formulations induce apoptosis and necrosis in human umbilical, embryonic, and placental cells. Chem Res Toxicol. 2009;22(1):97-105. 
33. Abu-Qare AW, Abou-Donia MB. Biomarkers of apoptosis: Release of cytochrome c, activation of caspase-3, induction of 8-hydroxy-2'deoxyguanosine, increased 3-nitrotyrosine, and alteration of p53 gene. J Toxicol Env Heal B. 2001;4(3):313-332.
34. Guelen L. Development of an Efficient and Tumor Specific Apoptosis Inducing System Using Viral Gene Products [PhD thesis]. London, UK: University of London; 2004 


\section{Supplementary information Construction of pJDK-apoptin plasmid DNA (pDNA)}

The apoptin gene was amplified by the polymerase chain reaction (PCR) with Taq DNA polymerase (SolGent, Daejon, Korea) from the pEGFP-C1-apo template resulting

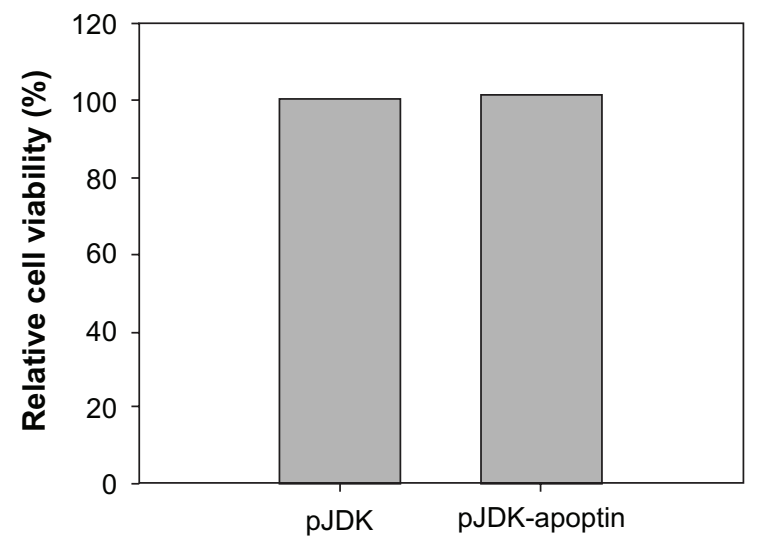

Figure SI Cell viability of normal brain cell line HT22.

in a $0.37-\mathrm{kb}$ PCR fragment, which was digested with and inserted between the EcoRI and $X b a \mathrm{I}$ sites of the pJDK vector together with the cytomegalovirus promoter. The PCR primers were 5'-CCCGAATTCATGAACGCTCTC-3' and 5'-GCCCTCTAGATCACAGTCTTATACGCCTTC- ${ }^{\prime}$. The DNA was sequenced to confirm the presence of the desired mutations and in-frame fusions. ${ }^{1}$ Supercoiled DNA was prepared from a culture of Escherichia coli $\mathrm{DH} 5 \alpha$ by using an Endofree Plasmid Maxi Kit (Qiagen, Valencia, CA, USA). The final DNA stock was dissolved in endotoxin-free Tris-EDTA (TE) buffer. The quality and concentration of the DNA were assessed by ultraviolet (UV) absorbance at $260 \mathrm{~nm}$ and $280 \mathrm{~nm}$ resulting in optical density ratios of 1.8-2.0. The DNA was stored at $-70^{\circ} \mathrm{C}$ until use.

\section{Reference}

1. Fischer U, Janssen K, Hofmann TG, Jans DA, Hay RT, Schulze-Osthoff K. Apoptin is modified by SUMO conjugation and targeted to promyelocytic leukemia protein nuclear bodies. Oncogene. 2007;26(11):1557-1566.
International Journal of Nanomedicine

\section{Publish your work in this journal}

The International Journal of Nanomedicine is an international, peerreviewed journal focusing on the application of nanotechnology in diagnostics, therapeutics, and drug delivery systems throughout the biomedical field. This journal is indexed on PubMed Central, MedLine, CAS, SciSearch ${ }^{\circledR}$, Current Contents ${ }^{\circledR} /$ Clinical Medicine,

\section{Dovepress}

Journal Citation Reports/Science Edition, EMBase, Scopus and the Elsevier Bibliographic databases. The manuscript management system is completely online and includes a very quick and fair peer-review system, which is all easy to use. Visit http://www.dovepress.com/ testimonials.php to read real quotes from published authors. 